used cause the termination of synthesis of the first of the three enzymes specified by the lactose operon and, moreover, prevent synthesis of the two subsequent genes in the mRNA even though these do not themselves contain mutations. In the revertant strains, the translation of the first gene is started, then terminated by the nonsense mutation and finally re-started by the new initiator site. As a result, although the first gene is translated not as a single functional enzyme molecule but as two pieces, the second and third genes of the $m$ RNA are translated to produce enzyme.

The initiator reversion can be used to determine the nature of the initiator sequence by constructing, in genetic crosses, a strain of bacteria in which the new initiator occurs before and not after the nonsense mutation. The only differences in the sequences of amino-acids in the polypeptide chains produced by the initiator-nonsense strain and the nonsense strain will be specified by the initiator sequence of codons. Thus it should be possible to work out whether the initiator is simply an AUG or GUG codon or a more elaborate sequence.

In the same issue of the Journal of Molecular Biology (page 413), Beckwith's group report yet another step in the elegant analysis of the lactose operon of $E$. coli. By deletion mapping, they have now confirmed their earlier suggestion that the order of the genes in the operon is repressor gene, promoter gene, operator gene and then the genes for the three enzymes- $\beta$ galactosidase, permease and transacetylase. Moreover, they have also shown that the promoter and operator genes are distinct, that the base sequence of the former determines binding of RNA polymerase while the sequence of the operator serves as a binding site for repressor protein. There may be some overlap, for strains lacking the promoter make very small amounts of the three enzymes, implying that RNA polymerase can bind weakly to the operator, although there is no doubt that in the main the two regions are distinct. The combined promoter-operator region is apparently only a small part of the operon; the two genes are very much smaller than average, perhaps only a hundred base pairs.

\section{ENZYMES \\ How Enzymes Work}

\section{from a Correspondent}

IT is more than three years since the crystallographic studies of Phillips and his colleagues revealed the complete three-dimensional structure of lysozyme. Other enzymes soon followed-chymotrypsin, ribonuclease, carboxypeptidase, papain-and the latest in the series is published on page 235 of this issue. 'The structure of the enzyme subtilisin $\mathrm{BPN}^{\prime}$ has been determined at $2.5 \AA$ resolution by Professor Kraut and his colleagues of the University of California. Enzymologists have on occasion been sceptical whether the static images of crystallography would ever suffice to interpret the transient events of catalysis. There was perhaps some justification for this pessimism initially: in no case did the plethora of new struetural information reveal unambiguously the details of function that were of prime interest to enzymologists. But recently X-ray studies have begun to yield results that should excite even the most hardened sceptic.
The recent work of Lipscomb and his associates on carboxypeptidase $A$ has been of enormous interest. They have shown that when a substrate, glycyltyrosine, binds to the active site of the enzyme, a widespread conformation change is induced which involves the movement of a tyrosine residue through no less than $14 \AA$. This is an important observation for several reasons. It is the first unequivocal demonstration of induced fit, the theory that the act of binding of substrate to enzyme induces a necessary alignment of catalytic groups on the enzyme surface. Furthermore, the sandwiching of the substrate beneath the hydrophobic flap of tyrosine, and on top of a charged zine atom, suggests a strategy of enzyme action, the pitting of electrostatic energies against hydrophobic energies, which may be of general relevance.

Professor Kraut's new work continues this theme in an unexpected way. Subtilisin $\mathrm{BPN}^{\prime}$ is an extracellular protease produced by Bacillus amyloliquefaciens: like chymotrypsin and trypsin its catalytic power depends on a reactive serine residue. Although subtilisin has no resemblance to chymotrypsin at the level of primary sequences, Professor Kraut's analysis of subtilisin reveals that, again like chymotrypsin, the reactive serine residue probably hydrogen bonds to a histidine residue. And it emerged at the Royal Society meeting on proteolytic enzymes in December that the chemical parallel between the two enzymes goes beyond this, for in both cases the active site histidine also hydrogen bonds by its distal nitrogen atom to a buried acid group, aspartate. It was announced at the same meeting that a third protease, pig pancreatic elastase, shares this pattern.

The negatively charged aspartate, embedded among hydrophobic residues, presumably alleviates its situation by a transfer of charge along the chain of hydrogen bonds via histidine to the serine in the active site at the surface of the molecule. And the serine residue in the active site is thereby made more nucleophilic. This was a revelation for even the most classically minded enzymologist: the hypothetical coupling, by hydrogen bonding, of serine and histidine at the active sites of enzymes has been a popular pastime for years, but always seemed dubious given the enormous gulf in acidity between histidine's imidazole and serine's aliphatic hydroxyl. Coupling of an imidazole group to a buried acid group will effectively lower its acidity, and facilitate its hydrogen bonding with the serine residuc.

Other features of subtilisin structure are less surprising. Like chymotrypsin, the active site does not lie in any particular cleft; like carboxypeptidase $A$, much of its core is in a pleated sheet structure; like papain, the backbone chain has points of definite overall folding, and the active site is situated at a point of convergence of different segments of the main chain. The side chain movements seen on sulphonylation of the reactive serine appear very similar to those in a-chymotrypsin.

There is every expectation that the crystallographers will keep up this pressure of new ideas about even quite familiar biochemical systems. Proteases have had more than their share of the spotlight recently, and it is good news that the structures of several dehydrogenases are in the pipeline and that even aspartate transcarbamylase, the touchstone of allosterism, shows signs of yielding to attack. 